\title{
Naïve Realism and the Colors of Afterimages
}

\author{
Vivian Mizrahi ${ }^{1}$ (i)
}

Accepted: 5 October 2021 / Published online: 16 October 2021

(c) The Author(s) 2021

\begin{abstract}
Along with hallucinations and illusions, afterimages have shaped the philosophical debate about the nature of perception. Often referred to as optical or visual illusions, experiences of afterimages have been abundantly exploited by philosophers to argue against naïve realism. This paper offers an alternative account to this traditional view by providing a tentative account of the colors of the afterimages from an objectivist perspective. Contrary to the widespread approach to afterimages, this paper explores the possibility that the colors of afterimages are not ontologically different from "ordinary" colors and that experiences of afterimages fail to provide a motivation for rejecting naïve realism.
\end{abstract}

\section{Naïve Realism and the Challenge of Afterimages}

Naive realism claims that what we perceive is a fragment of the world itself and that no mind-dependent entities, like sense-data or intermediate representations, are needed to explain the phenomenal character of perceptual experiences. This view has two great merits. First, naïve realism fits nicely with the phenomenology of ordinary perceptions. Perceptual experience is indeed phenomenally transparent to its objects in the sense that "introspection of one's perceptual experience reveals only the mind-independent objects, qualities and relations that one learns about through perception" (Martin 2002: 378). When I see a banana before me, the yellowness I perceive is experienced as a feature of the fruit. Many philosophers add that it is not experienced as a feature of my perceptual experience. But this may also be thought to be an inference from the first claim. The unmediated awareness of mind-independent objects and properties endorsed by naïve realists not only provides a way to characterize the relation between the perceiver and the perceived object, but also explains the phenomenal character of perceptual experiences. The second reason for naïve realism's appeal is epistemological. If there is no intermediary between the

Vivian Mizrahi

vivian@mizrahi.ch

1 Thumos Group, Campus Biotech, CISA - University of Geneva, Case Postale 60,

CH - 1211 Genève 20, Switzerland 
perceiver and the world, naïve realists can easily account for the foundational role of perception in knowledge. Unlike theories that make use of mind-dependent intermediaries to account for perception, the naiive realist's account does not introduce a "veil of perception" between the perceiver and the world and therefore avoids the trap of a wholesale skepticism.

Despite its phenomenological and epistemological merits, naïve realism is often said to face some serious difficulties. One of these is the possibility of illusions and perceptual errors. Indeed, if perceptual experiences are constituted ${ }^{1}$ only by mindindependent objects and properties, how can there be illusory or erroneous perceptual experiences? How can perceptual experiences be constituted by things that are different from what they really are? Naïve realism seems inadequate to explain illusion and misperception, because it does not acknowledge a dichotomy between what things are and how they appear.

Critics of naive realism have adduced a number of phenomena as counterexamples to unmediated access to a mind-independent reality. Such critics regard afterimages as particularly cogent in this respect, because they not only present chromatic properties that are not there to be perceived, but also seem to lack the kind of perceptual independence advocated by naïve realists. ${ }^{2}$

The word afterimage is commonly used to refer to optical phenomena that occur when the eyes are intensively exposed to a source of light or a static image. Although there are two major types of afterimages_-positive and negative- this paper will be limited to negative afterimages. Negative afterimages occur when subjects stare at a colored pattern for an extended period of time and then look at a white or lightcolored surface. Even though this surface is uniform, observers perceive a colored pattern on the surface similar in shape to the image at which they were previously staring but with different colors. When, for example, a subject stares at a green bird for $20 \mathrm{~s}$ and then looks immediately at a white sheet of paper, a faint magenta bird will appear on the sheet. Conversely, if a subject stares at a red parrot and then looks at a white surface, a blue-green parrot will appear. ${ }^{3}$

Afterimages are often referred to as optical or visual illusions, and their supposed illusory character has been exploited by philosophers to argue against particular theories of perception ${ }^{4}$ and particular theories of colors. ${ }^{5}$ Few philosophers, however, have tried to understand the nature of afterimages and to explain why they should count as illusions. ${ }^{6}$

\footnotetext{
${ }^{1}$ I endorse here a standard version of naïve realism (see e.g. Campbell 2002; Fish 2009; French 2014; Logue 2012; Martin 2002) which claims that the mind-independent items a subject perceives constitutively shape his/her perceptual experience. One may of course prefer to say that perception depends on its objects rather that it is constituted by these.

2 See Boghossian and Velleman (1989), Block (1996), Kind (2008).

3 These examples can be found at https://www.exploratorium.edu/snacks/bird-in-cage.

${ }^{4}$ See Moore (1942), Block (1996), Boghossian and Velleman (1989), O’Shaughnessy (2000), Siegel (2010).

${ }^{5}$ See Campbell (1979), Jackson (1977), Thompson et al. (1992).

${ }^{6}$ Notable exceptions are Churchland (2005) and Phillips (2013).
} 
This paper intends to partly fill this gap by investigating the nature of negative afterimages and by explaining how perceptions of afterimages differ from "normal" visual experiences but also from traditional cases of illusion and hallucination.

My discussion is structured as follows. First, I introduce afterimages and describe the special features which, according to many, make them differ from publicly visible objects. I also explain why those same traits seem to threaten naïve realism. I then introduce naïve realism and consider its implication for color perception and color ontology. I show, in particular, why I consider a certain form of color physicalism to be in perfect agreement with naïve realism. Finally, I argue that despite their special features it is possible to provide an account of the colors of afterimages according to which they are not different in nature from colors perceived in "standard" circumstances and which therefore avoids the threat they are supposed to constitute to naïve realism.

\section{The Phenomenology of Afterimages}

Experiences of negative afterimages are often considered illusions or even hallucinations. In view of their particular phenomenology, this is not really surprising. Experiencing negative afterimages indeed appears to differ from typical visual experiences in a number of ways. Unlike mundane objects, afterimages are ephemeral and private, and their perception does not seem to present the world as it really is. Let's consider each of these characteristics in turn.

\subsection{Ephemerality}

Unlike experiences of "moderate-sized specimens of dry goods," 7 like tables or trees, experiences of afterimages do not last very long. Like experiences of rainbows or "mirages" above hot surfaces, experiences of afterimages are short-lived. Their fluctuating and fleeting character is accompanied by a lack of substantiality which seems to prevent them from being anchored in space. ${ }^{8}$ The apparent lack of materiality of afterimages is reinforced by the way their size seems to vary according to the distance between the observer and the surface on which they are perceived. In contrast to common material objects, whose dimensions are unaltered by the subject's visual standpoint, the size of afterimages is directly correlated to the distance from the observer's eyes to the surface on which the afterimage is perceived.

Because experiences of afterimages contrast in many ways with the appearance of material objects, they are often used to challenge naïve realism, which supposes that physical properties of perceived objects explain the phenomenological properties of perceptual experiences. Because afterimages instantiate experiences of entities that

\footnotetext{
7 J. L. Austin 1962, p.20.

8 For a general presentation of the notion of "the ephemeral" in the philosophy of perception and a detailed discussion about its relation to the notion of "the insubstantial" rather than "the fleeting" or the "short-lived", see Crowther and Mac Cumhaill (2018).
} 
seem to lack the material properties that causally explain how an observer is perceptually connected to the perceived object, they suggest that perceptual experience cannot be accounted for solely in terms of a mind-independent reality.

\subsection{Privacy}

Afterimages seem to lack the materiality of concrete objects. They can be considered in this respect to be pure visibilia. ${ }^{9}$ like rainbows, shadows, and holograms. Although pure visibilia differ from material objects in many ways, their objective reality is rarely contested. Rainbows and shadows are immaterial and fleeting objects, but their existence is indeed accessible to any suitably positioned perceiver. The existence of afterimages is more controversial, because it seems that afterimages cannot be simultaneously perceived by different observers. Unlike rainbows or shadows, a particular afterimage can be experienced by one observer only. Different observers can experience similar or even qualitatively identical afterimages, but they cannot perceive a numerically identical afterimage. The private dimension of afterimages seems to contradict naïve realism, which claim that perceptual experiences must be understood as a direct relation to an objective reality. Because afterimages do not seem to be perceptions of publicly observable objects, it can be argued that they are purely sensational or perceptions of subjective entities. ${ }^{10}$ Although visible objects are not private entities, there is a wide variety of situations in which our perceptual experiences of external reality are difficult to share with others; this is particularly the case when observers have different visual systems. I will argue that experiences of the colors of afterimages are very similar to the experiences that characterize such situations. Unlike colors perceived in standard conditions, like the yellowness of a banana perceived in daylight, colors of afterimages are only accessible after some change in the visual system of the observer has occurred. As I will argue, the colors of afterimages are not private in the sense of being subjective, they are private only in the sense of being usually accessible to a limited number of observers only.

\subsection{Illusory Appearances}

According to naïve realism, if afterimages are perceptual experiences, they should present observers with mind-independent objects. But it is notoriously difficult to identify what these objects are. According to J. C. Smart (1963, pp. 94-95), for example, afterimages are visual experiences of a white screen covered by an illusory colored patch:

The man who reports a yellowish orange after-image does so in effect as follows: "What is going on in me is like what is going on in me when my eyes

\footnotetext{
9 See Martin (2010) for a discussion of pure visibilia.

${ }^{10}$ Cf. Boghossian and Velleman (1989), Kind (2008), O’Shaughnessy (2000).
} 
are open, the light is normal etc. etc. and there really is a yellowish-orange patch on the wall."

But is that really the way afterimages appear? If you've seen an afterimage, you've probably noticed that, contrary to a colored patch, an afterimage does not appear to belong to the surface on which it is perceived. Unlike the color patch on a wall, the afterimage's location depends on the movement of the eyes and not on its location on the surface. Moreover, unlike a real colored patch on a wall, afterimages cannot be explored by changing the position of our body. We cannot gain information about an afterimage by changing the distance, angle, or perspective we have in relation to it.

Illusions are considered partially veridical in the sense that an object is veridically perceived but not with its real properties. Thus Smith (2002, p. 23) applies "illusion" to any perceptual situation in which a physical object is perceived, but "in which that object perceptually appears other than it really is, for whatever reason".

So, if afterimages are not perceived as properties of the surface on which they are projected, it is difficult to specify what kind of illusion afterimages really are. That is, what the object is that appears to be different from what it really is.

Boghossian and Velleman (1989, p. 87) expressed a similar concern:

This problem would not arise if after-images were full-blown illusions. That is, if seeing an after-image consisted in seeming to see a material object suspended in physical space, then that object, though in fact illusory, could still appear to have the same colour quality as any other material object. But afterimages are not seen as material objects, any more than, say, a ringing in one's ears is heard as a real noise. The items involved in these experiences are not perceived as existing independently of being perceived.

Afterimages seem to present objects that are not there. An observer can, for example, see a colored bird even though there is nothing in front of him but a blank page. As Macpherson (2013, p. 8) maintained, afterimages seem to be cases of hallucinations rather than of illusions:

Another case worthy of note is that which is often called "having an afterimage." If one stares at a patch of color and then looks at a white surface, one has an inaccurate experience as of a patch of the same shape as the one stared at originally, but in the complementary color. Many philosophers would, I think rightly, count such cases as being cases of hallucination. If that is right then they are further instances of partial hallucinations, for one still sees the world when having an afterimage.

But if afterimages are classed as hallucinations, it is tempting to conclude that afterimages are not mind-independent objects and that they are essentially mental.

The view of afterimages defended in this paper goes in the opposite direction. It claims that afterimages are neither hallucinations nor illusions, but rather optical phenomena related to unusual experiences of colors. These experiences are neither illusory nor mistaken, and are not ontologically different from veridical experiences. 


\subsection{Reservations and Limitations ${ }^{11}$}

The view of afterimages I will defend in this paper is partial and incomplete. It focuses exclusively on the colors of afterimages. Is it not deeply misleading to limit the discussion of afterimages to the nature of their colors and thus to neglect their other properties, in particular, their spatial properties? This neglect, it may well be thought, leaves out just what makes afterimages peculiar in the first place. It can be argued, for example, that the colors of afterimages cannot be real colors because they are perceived as delimiting shapes which are not real, or at least not real in the same sense as the colored shapes we normally see in front of us. In this respect afterimages share many features with other optical phenomena such as double vision, eye floaters, blurry vision, etc.. A comprehensive study of afterimages would therefore require an investigation of their relations to these phenomena and an assessment of the implications of these relations for a realist view of perception. That is not a task that can be undertaken here.

\section{The Science of Afterimages}

Afterimages may seem to be magical or illusory, but the science behind them can be easily understood and mostly explained by photoreceptor (cone) cells adaptation. ${ }^{12}$ Here, then, are the facts. We perceive colors using cells called cone cells. There are three types of cone cells, each with different pigments: $\mathrm{S}$-cones, M-cones, and L-cones. Each cone is sensitive to visible wavelengths of light that correspond to short-wavelength, medium-wavelength, and long-wavelength light. The color yellow, for example, is perceived when the L-cones and M-cones are stimulated more than the S-cones, and the color red is perceived when the L-cones are stimulated significantly more than the M-cones. Similarly, blue and violet hues are perceived when the S-cones are stimulated more than the other two. ${ }^{13}$

If you look at one color for a very long time, the corresponding cone cells can become fatigued and, as a result, temporarily fail to respond. As long as this failure

\footnotetext{
${ }^{11}$ My thanks go to an anonymous referee of this paper for pointing out the restrictive scope of this paper and some of the possible consequences of the decision to limit the scope of this paper to the colors of afterimages.

12 The full mechanism that produces afterimages is however complex and not fully understood. The question whether the retinal process alone or retinal and cortical processes jointly cause afterimages is still intensively discussed. It is important to stress however that all recent proposals (those invoking purely retinal and those appealing to the cortex) rest on the fundamental observation that a reduction in the sensitivity of retinal photoreceptors constitutes the main mechanism behind the formation of afterimages. See MacLeod and Hayhoe 1974, Zaidi et al. (2012), Shevell et al. (2008), Shimojo et al. (2001).

Besides the cones which detect light entering the eye, color perception involves numerous other mechanisms. Excitations of the cones initiate the receptoral level of color vision, but the cone signals are then propagated to postreceptoral levels where those signals are combined and processed.

${ }^{13}$ Besides the cones which detect light entering the eye, color perception involves numerous other mechanisms. Excitations of the cones initiate the receptoral level of color vision, but the cone signals are then propagated to postreceptoral levels where those signals are combined and processed.
} 
lasts, fatigued cone cells lose their sensitivity, while the other cone cells remain operative. After several seconds, the fatigued cones will recover. If you stare, for example, at a red picture and immediately look at a white area afterwards, you will see a greenish afterimage that has the same shape as the red picture. This is because staring at a red surface fatigues the L-cones and reduces their sensitivity. As a result, a white surface that stimulates all cones roughly equally in normal situations will stimulate $\mathrm{S}$-cones and $\mathrm{M}$-cones more than L-cones, causing a greenish image to be perceived. The same explanation goes for the other colors: staring at a blue-green image will make a reddish image appear on a white surface, staring at a yellow image will make blueish image to appear, etc. ${ }^{1415}$

Although these facts can help us understand what causes subjects to see afterimages, they do not seem to explain why the colors of afterimages are apparently so different from real colors, like the yellowness of bananas or the redness of ripe tomatoes. The next step is to show that, although the colors of afterimages are different from standard colors, in a way to be explained, they are nevertheless bona fide colors and are not ontologically different from the yellowness of bananas or the redness of ripe tomatoes. But before I can make that argument, an account of the nature of colors is needed.

\section{Naïve Realism and the Nature of Colors}

Naïve realism holds that perceptual experiences are constituted by mind-independent objects and properties. As we have seen, the naïve realist's view does not admit a dichotomy between appearance and reality. The naïve realist is therefore committed to a realist view of perceptual qualities, which holds that objects have the perceptual qualities they appear to have. In the case of colors, this means that whatever color $\mathrm{C}$ one perceives an object $\mathrm{O}$ to be, $\mathrm{O}$ is $\mathrm{C}$.

At first glance, this view is highly implausible, because objects often present conflicting appearances with respect to colors. Snow usually appears to be white, but it can appear yellow when perceived through ski goggles or pink when observed at dusk. If there is no dichotomy between appearance and reality, it seems that the naive realist must claim that snow is yellow when perceived through ski goggles and pink when perceived at dusk-a view that apparently conflicts with the commonsense affirmation that snow is white.

\footnotetext{
14 As pointed out to me by an anonymous referee, afterimages can occur with closed eyes. Because there is no direct cone stimulation, experiencing afterimages with closed eyes suggests that cone adaptation alone cannot account for experiences of afterimages. I acknowledge the difficulty this poses for the argument developed here, but I believe the problem of afterimages with closed eyes should be considered as an aspect of a more general problem regarding the status of what is perceived or not perceived in darkness. See Sorensen (2008) and Wright (2012).

15 There are other forms of afterimages (presumably cortical) which are perceived when rapidly altering patterns of light elicit colors. A retina-only explanation seems to be insufficient in those cases. See Virsu andLaurien (1977) and Shimojo S, Kamitani Y, Nishida S. (2001).
} 
In fact, as stressed by some philosophers, ${ }^{16}$ such a conflict arises only if the naïve realist subscribes to color monism, which holds that a uniformly colored surface can have at most only one color. Implicit in most color theories, color monism is problematic for the color realist, because there seems to be no way to determine which color among all the color appearances is the real color of a surface. The problem introduced by this approach is clearly presented in Berkeley's first dialogue between Philonous and Hylas:

[E] ven when all the other factors remain unaltered some objects present different colours to the eye depending on the angle from which they are looked at. The same thing happens when we view an object in different brightnesses of light. And everyone knows that the same bodies appear differently coloured by candlelight from what they do in daylight. . .. Now tell me whether you still think that each body has its true, real colour inhering in it. If you think it has, I want to know what particular distance and orientation of the object, what special condition of the eye, what intensity or kind of light is needed for discovering that true colour and distinguishing it from the apparent ones. (Berkeley 1734)

The problem with color monism is not so much that we don't know how to single out the real colors, but rather that any attempt to identify colors with one particular type or family of properties detected by the human visual system appears to be arbitrary. If we favor, for example, human trichromatic perception over the perception of dichromatic (color-blind) subjects because dichromatic subjects have a reduced ability to discriminate colors, we should also favor tetrachromatic color perception, the color perception possessed by birds, over human color perception-and conclude that no human has ever seen the true colors of objects. Color monism leads straightforwardly to a massive-error view of color perception, which holds that we are victims of systematic and pervasive error about the color properties in the world. To avoid the problems involved in the high degree of variability exhibited by color perception, the naïve realist must reject color monism and embrace a pluralist view of colors, a view which admits that a colored surface has many different objective colors. ${ }^{17}$ The point is nicely formulated by Kalderon (2007, p. 584): "The world may be as it appears to be; it is just that it is more than it appears as well."

The rest of this paper will show that color pluralism fits perfectly with naïve realism and explains the colors of afterimages, as well as other color phenomena.

\section{Reflectance Physicalism and Color Pluralism}

The pluralist view of colors I will assume in this paper is both objectivist and physicalist in the sense that it supports the view that colors exist independently of our perception of them and that they are identical to certain physical properties of

\footnotetext{
${ }^{16}$ See Kalderon (2007) and Mizrahi (2006)

${ }^{17}$ Color pluralist views have been defended in Matthen (1999), Mizrahi (2006), Kalderon (2007), and Allen (2009).
} 
objects. Like other physicalist approaches to colors, the view I propose does not restrict colors to the colors perceived by standard human observers. Although the physical properties identified with colors must causally explain human color perception, they must also explain color-vision differences among human subjects and across species. A similar approach to color physicalism can be found in Byrne and Hibert (Byrne and Hilbert 2003, p. 57), who argued that "there is no incompatibility between our version of physicalism and the thesis that many nonhuman animals have color vision." The present proposal offers a distinctive characterization of color variations across illuminants and, as I believe will become clear later on, provides an original approach to the colors of afterimages and some other interesting related phenomena. Unlike most objectivist and physicalist accounts of colors, the present proposal does not favor one illuminant, or one type of illuminant, over others. In particular, it does not assume that natural daylight or any other entire-spectrum light source is preferable for determining an object's real color. ${ }^{18}$ It can surely be argued that an entire-spectrum light is superior for some tasks, but it cannot be concluded from this fact alone that illuminants that do not emit light continuously across the entire visible spectrum cannot give us access to an object's real color. Color physicalists who identify colors with surface spectral reflectances (SSRs), or with reflectance types, tend to assume that only an entire-spectrum illuminant can be used to perceive an object's real color. Because SSR is the proportion of incident light a surface is disposed to reflect at each wavelength in the visible spectrum, they sensibly argue that entire-spectrum illuminants are required to discriminate between SSRs and therefore to perceive colors. However, this approach is misleading. If reflectance physicalists are unwilling to arbitrarily restrict the capacity to perceive colors to humans, and because many species can see frequencies of light that cannot be detected by human color receptors, reflectance physicalists have to extend the visible spectrum to wavelengths invisible to the human eye. Yet, extending the notion of "visible light" to frequencies that cannot be perceived by humans has several important consequences. First, if SSR is defined as the proportion of light that a surface reflects at each wavelength in the visible spectrum of any species, and because colors are in this case identified with physical properties which cannot be detected by the human visual system, human observers can strictly speaking never perceive colors. Moreover, it would not help to identify colors with reflectance types instead of SSRs, as proposed by Byrne and Hibert (Byrne and Hilbert 2003). We do, in fact, know that, unlike humans, many animals have color receptors sensitive to UV light. Yet the capacity to perceive reflectance relative to UV light can make a huge difference in terms of the colors perceived. We know, for example, that some flowers exhibit UV patterns created by UV color discontinuities, discernible by insects but invisible to humans. ${ }^{19}$ Because humans perceive such petals as uniformly colored, there is at least one color they cannot perceive due to their limited spectral sensitivity. Now, what should reflectance physicalists, such as Byrne and Hilbert (2003), say about the uniform color of petals perceived

${ }_{18}$ For a defense of natural daylight as determining the real colors of objects, see Allen (2010).

19 Knuth (1891). 
by a normal trichromat viewer? Is this color real, or is it only apparent? If it is only apparent, it seems that reflectance physicalists are committed to the view that ordinary color experience involves massive error-a view which is incompatible with all the epistemological and ontological virtues of color physicalism. If the uniform color perceived by a normal trichromat viewer on the surface of the petals is real, reflectance physicalists must accept that the types of reflectance that can be identified with colors should not be restricted to reflectances associated with entire-spectrum illuminants. In fact, what the UV color vision of insects reveals is that there is no nonarbitrary way to choose between illuminants.

The case for illuminant pluralism can also be made through the example of metamerism. Surfaces that have different spectral reflectances but match visually under a given illuminant for a given observer are said to be metamers for that illuminant and that observer. Given their different spectral differences, metamers under a given illuminant will not appear to match under some other illuminant. For most observers and activities, color comparisons are done in some form of white light (daylight or artificial light). However, for particular laboratory or industrial purposes, the relevant illuminant may be composed of different bands of wavelengths or even a unique wavelength. For example, metameric inks, which match in "normal" light conditions, can be used in security applications. Using this technique, a printer can conceal a word, message, or image, which is invisible to the human eye until the lighting conditions change. The same technique is also used in bank-note printing to prevent counterfeiting. Reflectance physicalists, who single out entire-spectrum illuminants as revealing the real colors of things, have to deny that chromatic discontinuities perceived under narrow-band light sources are real. They must therefore conclude, against common sense, that visual experiences in which pieces of evidence or hidden messages are detected by using particular light sources are illusory, because the colors perceived under such illuminants are only apparent. But this odd conclusion has no obvious justification, except perhaps a practical preference for entirespectrum illuminants. The use of narrow-band light sources does in fact reduce our discriminatory capacities in everyday life, because differences of reflectance relative to a few wavelengths are much less numerous than differences of reflectance relative to many wavelengths. This simple fact is sufficient to explain why "white" lights are usually preferred for color perception and object recognition. But from an ontological point of view, there is no reason to favor "white" lights over narrow-band or single-wavelength illuminants.

Another reason for extending veridical color experiences to color perception with different illuminants is more general and, I believe, more profound. The high degree of variability in the colors exhibited by any surface under different illuminants constitutes an important characteristic of colors and color perception. But most philosophical theories of color have ruled out these variations by drawing a distinction between real and apparent colors.

But why should one assume that systematic chromatic changes due to illuminant variations are only apparent? Are reflectance physicalists really willing to set aside all color variations due to illuminant variations as illusory because they do not involve SSR variations? Is the greenness of a banana under a "blue" light not as 
fundamental for understanding colors as its yellow appearance in daylight? Is the pink shade of snow at dusk not a real chromatic phenomenon worth explaining? More generally, would our knowledge of colors be the same if all these variations were absent from our experience? I doubt it. Color variations are diverse. We can notice the maturity of a piece of fruit by noticing the color change of its skin, but we can also notice changes in atmospheric properties by noticing a transient change of a meadow's color. Those color variations are different in nature, but why should we not consider them to be equally real $?^{20}$

\section{Color Selectionism}

Color pluralism is the view that objects have simultaneously different colors. When combined with a physicalist theory of colors, color pluralism allows objects to simultaneously have a plurality of mind-independent colors that can be perceived differently by different observers with different perceptual systems. As rightly pointed out by Kalderon (2007, p. 594), color pluralism goes hand in hand with selectionism:

The possibility of color pluralism may undermine the explicit argument for color relativism, but it is the selective nature of color perception that addresses the modal puzzlement that motivates Protagorean relativism and Democritean eliminativism alike. Selectionism provides an interpretation of the dependency of color appearances on the visual system of the perceiver that is consistent with color appearances being manifestations of mind-independent qualities of material objects.

According to color pluralism, conflicts in color appearances do not occur because colors are mind dependent, but rather because each particular visual system perceives only a fraction of the plurality of the existing mind-independent colors. Selectionism is traditionally associated with inter-personal variations, but few have stressed its role when trying to explain apparent conflicts between color appearances caused by lighting conditions. Yet there is a profound connection between color visual systems and lighting conditions. As we have seen, color visual systems differ according to the region of the spectrum to which they are sensitive and to the number of their color-receptor types. These physiological differences among visual systems explain in large part why different species, and even different observers, perceive different colors. Yet, color receptors resemble spectral filters in the sense that each type of photoreceptor is defined by a pigment that absorbs certain wavelengths

\footnotetext{
${ }^{20}$ Zaidi (2001, pp. 192-193) made a similar case by stressing the limits of color constancy:

"A large number of experimental studies have all concluded that object colors as measured by asymmetric matching are not perfectly constant across illuminants. I have previously proposed that it is better to ask new types of questions: Do materials appear to be of "systematically" different colors under different illuminants? Instead of viewing a failure of constancy as a limitation of the visual system, should it be regarded as a design feature that allows the observer to extract information about illuminants as well as objects?"
} 
of light better than others. From this perspective, there is no ontological difference between the selective process performed inside the visual apparatus by the cones and the selection of a particular range of light using chromatic filters. ${ }^{21}$ As brilliantly demonstrated by Akins (2014, pp. 181-183), it is possible to turn trichromats into monochromats by restricting the illuminant to a narrow spectral band of light:

In the RGB exhibit, the normal trichromat observes a room illuminated by one highly filtered light source, by the red, green, or blue light. Here, visible light is limited to an artificially small window by the filtered light source. That is, for us as trichromats, visible or "effective" light ranges from 370 to $660 \mathrm{~nm}$, a spectral range of roughly $300 \mathrm{~nm}$. But under the filtered lights of the exhibit, all light within the room is restricted to a narrow band of light about $60 \mathrm{~nm}$ in width. For the trichromatic viewer, then, spectral bandwidth is restricted by the sender not the receiver. Under the red, blue or green lights, whatever the trichromat sees is made visible by a single narrow spectral band of light, be it red, blue or green, reflected from the surfaces within the room. In effect, then, trichromatic observers have reduced spectral range very much like the restricted range of the rod achromat. In fact, one can think of the three lights as producing (very roughly) functional monochromats, each with only a short (blue), medium (green) and long (red) cone/photoreceptor.

The physicalist's strategy of accounting for color variations among perceivers by resorting to selectionism should therefore be extended to color variations caused by illuminants for the same reasons. Although colored surfaces instantiate a plurality of colors, the illuminant selects (where this means just that it determines a selection) which of these colors are perceived by a given observer.

The approach taken by most reflectance physicalists centers on the notion of SSRs, that is, the dispositional properties of surfaces to reflect a determinate amount of the incident light at each wavelength in the visible spectrum. Yet, as $\S 5$ shows, SSRs cannot be the physical properties detected by the human visual system, because it cannot discriminate between all the wavelengths constituting full-spectrum light. Moreover, if colors were restricted to SSRs, color vision would be limited to perception in full-spectrum light, which could be the case only if we arbitrarily restricted the notions of visible light and veridical perception. But SSRs are not the only reflectance properties of surfaces. A surface's reflectance property corresponds to the way a surface reflects the incident light, but its reaction to the light depends on the wavelengths entering into the composition of the incident light. This is why a blue surface on a white background that reflects a large proportion of short wavelengths included in white light (i.e.

\footnotetext{
${ }^{21}$ It is possible to say that photoreceptors on the retina operate like filters because they selectively transmit only a portion of the wavelengths contained in the entire light spectrum. A similar process can be found in digital cameras where tiny color filters are used to cover photosensors. In both cases, color information is captured by three types of photoreceptors or sensors with different absorption profiles which filter the light in a specific way.
} 
light source that approximates a uniform spectral power distribution) will reflect almost no light and appear almost black when illuminated with filtered light composed exclusively of long wavelengths.

There is not a unique way for a surface to interact with light, because light is not a simple and unique phenomenon. By decomposing light into rays of different wavelengths, Newton demonstrated that white light, though apparently simple, is in fact complex. Although light is not visible (Chisholm 1957; Heider 1959; Smart 1963; Hilbert 1987), the complexity of light is directly related to the variety of the colors we perceive. To grasp the importance of this relation, consider what our perception of colors would be like if light were simple and could vary only in intensity. If light were uniform, each point of a surface would reflect a determined proportion of the illuminant, but there would be no differences related to wavelengths. Provided that they reflect the same proportion of light, red, green, blue, and yellow surfaces would therefore be indiscriminable. Without the complexity of light and the diversity of illuminants, all phenomenological properties associated with color perception would vanish, because it is only through the interaction of surfaces with various wavelengths that the diversity of the intrinsic properties of surfaces can be accessed.

Unlike most objectivist and physicalist accounts of colors, my proposal does not favor one illuminant, or one type of illuminant, over others. In particular, it does not assume that natural daylight or any other entire-spectrum light source is preferable for determining an object's real color. It can certainly be argued that entire-spectrum light is superior for some tasks, but it cannot be concluded from this fact alone that illuminants that do not emit light continuously across the entire visible spectrum cannot give us access to an object's real color. According to this account, numerous colors can then be perceived in the absence of most wavelengths constituting the visible spectrum. In fact, as it appears, light composed of any combination of wavelengths -including single wavelength lights - projected onto a white surface will give rise to characteristic color experiences. ${ }^{22}$ None of those colors can be identified with SSR, because a surface's disposition to reflect a characteristic proportion of light at each wavelength cannot be accessed in the absence of those wavelengths. SSR cannot be perceived in the absence of entire-spectrum light, but all colored surfaces have stable dispositions to reflect different lights beside SSR. In fact, for any illuminant and any particular surface, there is a characteristic proportion of the incident light that a surface is disposed to reflect.

Traditional reflectance physicalism rightly identifies colors with dispositional properties of surfaces to interact with light, but it neglects two basic facts: light is not a single and uniform phenomenon, and each surface has as many reflectance properties as there are illuminants of different natures. Although all reflectance

\footnotetext{
22 Notice that the colors perceived in the absence of most wavelengths are typically the colors used in colorimetry to quantify and physically describe human color perception. In effect, color matching functions for a standard observer are obtained by mixing lights with a single wavelength. Cf. CIE (1932), Commission Internationale de l'Eclairage proceedings, 1931. (Cambridge: Cambridge University Press).
} 
properties are intrinsic and mostly stable properties of surfaces, they are accessible only under particular illuminants.

\section{The Nature of Afterimage Colors}

I believe that the failure to account in objective terms for the chromatic variations related to variation in lighting conditions has paved the way for many subjectivist views on color. By considering color variations produced by changes in the illuminant as merely apparent or illusory, philosophers have restricted real colors to a set of physical properties too constrained to give a full account of the complexity and richness of chromatic vision. Consequently, numerous color experiences have been discounted as mere illusions or devoid of any objective content. This is precisely the case with afterimage colors, which are notoriously experienced as phenomenologically different from the colors we usually perceive in daylight. Unlike colors perceived in standard conditions, the colors of afterimages were described by Churchland (2005), p. 545) as "chimerical":

This provides you with an experience of what might be called a chimerical color-a color that you will absolutely never encounter as an objective feature of a real physical object, but whose qualitative character you can nonetheless savor in an unusually produced illusory experience.

Yet, I believe that the unusual appearance of afterimage colors is not the result of their illusory nature but rather of their specific nature and of the specific nature of the human visual system. We indeed rarely encounter afterimage colors, because they are usually inaccessible to us. In what follows, I explain why perceiving such colors is rare and in what special circumstances they are perceived.

I have argued that perceiving a particular color depends both on the spectral sensitivity of color receptors and the spectral properties of the illuminant. To understand more deeply the connection between the sensitivity of color receptors and the nature of the illuminant, we must first recognize that changing the type of light source is not the only way to select and obtain illuminant color variations. The use of filters, for example, can produce color variations in different ways. First, filters can be used to modify the properties of the illuminant by selectively absorbing some of its wavelengths. By changing the properties of the illuminants, filters can therefore change the colors we perceive.

But filters can also be positioned between an observer and a colored surface. In this case, they do not directly filter the light emitted by the source but rather partially absorb the light reflected by the colored surfaces. For example, if a white surface, reflecting all the wavelengths equally, is viewed through a filter, only the wavelengths that are not absorbed by the filter will reach the eyes of the observer and be perceived. The observer perceives the same surface color in both cases. Whether the filter is located at the light source or between a reflecting surface and the observer, the way its transmitting properties select which reflectance properties are perceived by the observer is the same. 
To understand this point, consider two scenarios: a normal trichromat perceiver with his/her naked eyes observes a surface illuminated by a long-wavelength light (Fig. 1), and a normal trichromat perceiver with "red"-tinted glasses observes a surface illuminated by a white light (Fig. 2). Although the scenarios differ greatly, the reddish color of the surface experienced by the subject in both cases is identical with a unique property. In effect, the red-tinted glasses used in the second scenario are filters that block the medium and short wavelengths. Wearing such glasses therefore confines chromatic discriminations to the long-wavelength band. The use of a light composed exclusively of long wavelengths would have quite the same effect. The absence of short and medium wavelengths in the light would prevent color vision from operating in the short- and medium-wavelength band and therefore restrict chromatic perception to the long-wavelength band.

In a certain sense, the case of afterimages resembles to the scenario with an interposed filter, since the optical properties of the eyes are changed by selective fatigue. ${ }^{23}$ When the eyes are fatigued after a long exposure to a colored picture, they lose their sensitivity. If the L-cones are fatigued by staring at a red image, for instance, their reaction to long wavelengths will decrease, and consequently only the $\mathrm{S}$ - and M-cones will react to light, making a white surface appear blue-green.

Selective fatigue, acting like color filters, ${ }^{24}$ will therefore locally change the sensitivity of the retina. Instead of seeing the whiteness of the screen, fatigued cones will perceive its redness or blueness as though color filters had been interposed between the observer's eyes and the screen. ${ }^{25}$ Seeing an afterimage is then similar to seeing a photographic slide or, more precisely, seeing through a photographic slide. ${ }^{26}$ If this account is on the right track, it explains why the afterimages follow our gaze when we move our eyes and why their colors change according to the reflectance properties of the surfaces seen. Unlike chromatic changes due to ambient lighting, chromatic changes due to selective fatigue affect only a limited portion of the observer's visual field. As stressed by Phillips (2013), the way afterimages appear on a screen is similar to the way spots of light appear on a screen when "projected from where

\footnotetext{
${ }^{23}$ The hypothesis of the present proposal is that chromatic changes related to changes occurring in the retina can be accounted for in objective terms. However, some recent studies suggest that afterimage formation can be affected by perceptual and cognitive factors in addition to the bleaching of retinal photoreceptors. Although these findings are important for a better understanding of afterimage formation, it is beyond the scope of this paper to assess the putative consequences of these studies for the objectivist view it defends.

${ }^{24}$ It can be argued that my description of cones as functioning like filters is misleading because their sensitivity is not linear and that some cases of color constancy seem to conflict with this characterization. If this is the case, a better characterization must be provided. Meanwhile I suggest to keep this characterization as a useful heuristic tool aimed at uniting different visual media which selectively transmit only a portion of the wavelengths contained in the entire light spectrum.

${ }^{25}$ One consequence of the present account is that filters, like all transparent objects are colorless. In contrast to Katz's (1935) and Byrne and Hilbert's (2003) accounts, I believe a distinction between surface and voluminous colors is erroneous and that colors are superficial properties only. As argued in Mizrahi (2010), apparently voluminous colors are in reality surface colors perceived through transparent - and colorless - objects.

${ }^{26}$ As stressed in $§ 2.4$, the view of afterimages defended in this paper is only partial, because it focuses exclusively on the colors of afterimages. The view presented here must therefore be considered as exploratory in the sense that its scope is limited and its conclusions not decisive.
} 
Fig. 1 Perception in long-wavelength light

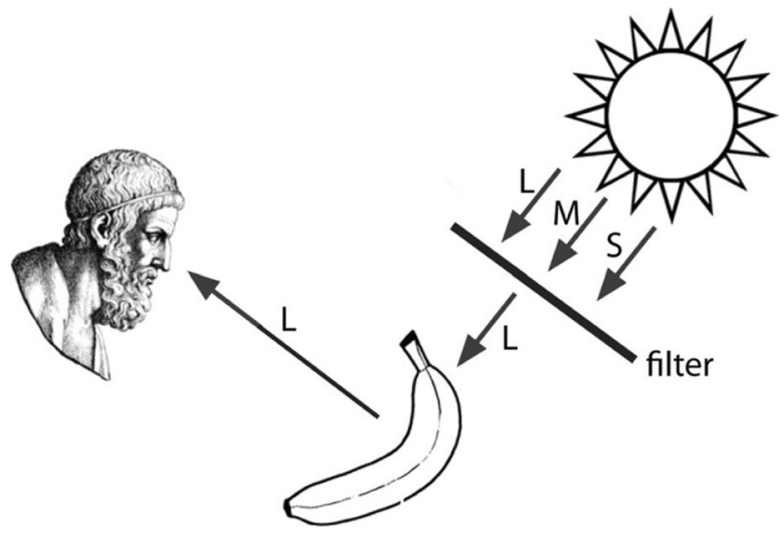

Fig. 2 Perception through a "red" filter

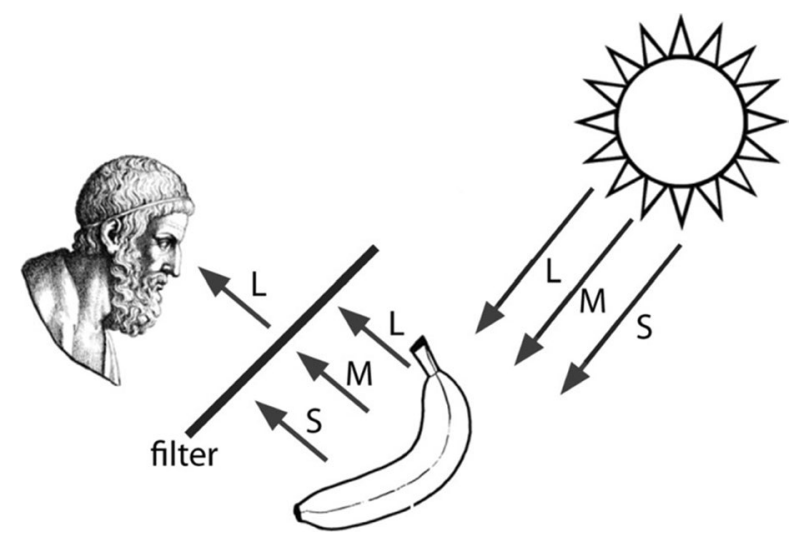

we are located". ${ }^{27}$ This similarity can be accounted for by the fact that the kind of chromatic changes caused by selective fatigue and projected spots of light are in both cases limited to a portion of the visual field. This explains in particular why we perceive a moving spot of light when a flashlight is swept across a homogeneous background and why we perceive an afterimage at different locations by moving our eyes.

According to the pluralist view of colors presupposed here, the colors of afterimages are real colors even though their perception occurs less frequently than the perception of colors in daylight without cone fatigue. This pluralist approach to afterimage colors can, moreover, offer a plausible account of their phenomenological idiosyncrasies. In order to complete my positive account of the colors of afterimages, I shall consider in turn these singularities.

${ }^{27}$ Phillips 2013, p.433. 
Unlike standard colors, colors of afterimages appear to be fleeting and transient. Although this appearance seems at first to be incompatible with their being colors of public and stable objects, I have argued that the colors of afterimages are objective and that they do not differ ontologically from standard colors. What explains their fleeting and transient character is the fact that they are caused by the selective desensitization of photoreceptors, a phenomenon which doesn't last long. The fleeting and transient character of the colors of afterimages does not therefore result from their special nature but rather from the from the temporary character of the cone fatigue that provide us with an awareness of these colors.

Consider next the appearance of afterimages as pure visibilia. If the kind of color shifts involved when perceiving afterimages is different from color transformations involving a chemical or physical change affecting the surface of a material object, it is because color shifts involved in afterimages result not from changes on the surface of colored objects but rather from the way the visual system selects which color is perceived. Unlike chromatic discontinuities due to physical discontinuities of a surface-like the different colors of a multicolored beach ball, which correspond to differences in the physical properties of its surface-the differences in color exhibited by afterimages or the projection of light of different wavelengths on a wall are not due to any physical discontinuities of the wall's surface. Those color differences correspond to colors made visible by using light of different wavelengths or by changing the sensitivity of the eyes. The surface of a wall can then appear to be of different colors without any discontinuities in the physical properties of its surface. If afterimages do not appear as a colored patch on the wall, as described by Smart, ${ }^{28}$ it is not because the perceived colors are not real or mind dependent, but simply because the kind of contrast they reveal does not correspond to the color contrasts exhibited by a colored patch on a wall. ${ }^{29}$

Now consider the claim that afterimages are private and therefore cannot be explained by public qualities of physical objects. Unlike most visible objects, such as chairs, trees, or even rainbows, afterimages are not the sort of thing we can point towards in order to share or compare our color experiences with each other. Experiences of afterimages are comparable in this regard to experiences of eye floaters ${ }^{30}$ or blurry vision: their phenomenological properties do not seem to be exhaustively characterized by what is perceived. It seems that there is something about these experiences that cannot be accounted for by the observer's awareness of the external world.

There are many ways to respond to the challenge raised by these cases, and it is beyond the scope of this paper to give a full answer to the challenge. I believe,

\footnotetext{
28 Cf. $\S 3$.

29 The present proposal predicts that if the changes to the sensitivity of the eyes were not short lasting and extended to the entire visual field, the colors perceived in afterimages experiences would lose their appearance as "visibilia" and turn into ordinary surface colors. Thanks to an anonymous reviewer of this journal for pointing this out to me.

${ }^{30}$ Eye floaters are spots in vision that appear like strings or cobwebs that drift about when you move your eyes. Eye floaters are generally caused by age-related changes that occur as the vitreous inside the eyes becomes more liquid.
} 
however, that the realist view of afterimage colors I have proposed indicates what such an answer might look like. What makes afterimages difficult to share with others is not the fact that they present private objects or properties but rather the fact that what is accessible to an observer is related to his/her visual system. I have argued that each visual system selects the colors it can perceive. The fact that different visual systems can perceive different colors explains why color perception may differ from one subject to another but also why those different visual systems can nonetheless all be correct. The case of afterimages is a little more complex, but it follows the same general lines. If the phenomenology of afterimages is unusual, it is not because their colors are only apparent or illusory, but because the changes in the sensitivity of the visual system caused by selective fatigue change and modify locally what colors are perceived. Since perceiving the colors of afterimages rests on modifications of individual visual systems, it may seem that these colors are private. What is, in fact, private are not the colors themselves, but the particular changes that explain these particular experiences. The kind of color experience exhibited by afterimages is very singular and therefore difficult to share with others. Enjoying afterimages is indeed often a solitary experience, unless other observers are willing to undergo the same physiological changes. In that case, it is possible for multiple people to perceive the same unusual colors, as when people sharing a pair of tinted sunglasses perceive snow as pink rather than white.

The approach to the colors of afterimages presented here bears many similarities to the view expressed in Phillips (2013) but there are also important differences. I consider first the similarities. Both accounts claim that appearances of afterimages can be exhaustively characterized by reference to mind-independent objects and properties. Moreover, they both stress a kind of affinity between the colors exhibited by afterimages and the colors perceived under projected lights. ${ }^{31}$ In contrast to most philosophical accounts of afterimages, which have maintained that afterimages reveal the existence of nonintentional entities like visual sensations, Phillips argued that afterimage experiences are not purely sensational because "they are indiscriminable from a certain kind of veridical perceptual experiences, namely an experience in which we encounter a certain kind of light phenomenon projected from our own perspective." (Phillips 2013, p. 433).

Like Phillips, I argue that the philosophy of perception has often suffered from an overly restricted "diet of examples" (ibid., p. 418). The ubiquitous examples of perceptual experiences of matte, opaque, uniform, and static objects in natural light have in fact marginalized an entire range of visual experiences. As a result, the more a perceptual experience departs from this restricted set of visual appearances, the more philosophers are tempted to tag it as subjective or purely sensational in nature.

The main divergence between our views has to do with the ontological status of afterimages. According to Phillips, afterimages are illusions of light phenomena,

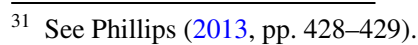


whereas in my view they are only special, and very unusual, ways to visually experience objective aspects of the world.

\section{Conclusion}

Like the colors selected by light of a particular wavelength, the colors of afterimages are neither illusory nor mental. They correspond to colors we don't usually see with the naked eye but can perceive under light of a certain wavelength or by using optical filters. The colors of afterimages reveal the fact that our visual system is a perceptual medium that selects the colors-real colors-we can see. Like optical filters, transparent air, or water, our eyes are causal intermediaries in the causal processes involved in vision. Although they give us access to reality, it's a limited access only. ${ }^{32}$

Color pluralism explains the colors of afterimages by stressing the role of the visual system as a selector. According to color pluralism, the colors of afterimages do not constitute a threat to naïve realism, because the constitutive relation of colors to color experiences is always guaranteed, even when color appearances seem to conflict with standard color experiences. As with other cases of misleading appearances, it can be argued that experiencing negative afterimages does not involve perceptual errors or illusions, even when they give rise to false beliefs or inappropriate behaviors. ${ }^{33}$ In fact, perceptual judgments rely not only on what is accessible through perceptual experiences but also on a rich contextual and sensorimotor knowledge. For example, determining whether an object is stationary or moving relies on the perceiver's implicit knowledge of his own movements as well as on what is perceived. Alva Noë (2004, p. 127) rightly stressed the predominant role of this background knowledge in color judgments:

Perceivers are in general implicitly familiar with the way apparent color varies as we move with respect to what we look at, or as other color-critical conditions change (e.g., changes in the character of ambient light, or in the colors of contrasting objects, etc.). Perceivers implicitly understand the patterns governing this sort of variation, just as they implicitly understand the way that the apparent shape of an object changes as they move in relation to the object.

The colors of afterimages are not different in nature from standard colors, but the highly unusual conditions of their perception prevent the perceiver from exploiting the implicit knowledge he would need in order to correctly interpret his perceptual experience. As a consequence, the sudden appearance of a colored patch on a white surface after selective fatigue gives the impression of having little to do with standard

\footnotetext{
32 As stressed in $\$ 2.4$, the view of afterimages defended in this paper is only partial, because it focuses exclusively on the colors of afterimages. This limited focus imposes important restrictions and imposes therefore some limitations on the conclusion presented here.

33 See Fish (2009), Genone (2014) and Mizrahi (2019).
} 
perception of colored surfaces, even though the same color could have been perceived by interposing a colored filter or projecting a spot of colored light onto a screen.

According to the view presented here (a view open to the objection mentioned in 2.4, which have not been replied to here), the colors of afterimages are not bizarre colors - they are not different in nature from the objective colors we can see around us. What is bizarre, however, is the context of their perception. In the levitatingwoman stage trick, the magician doesn't need to present a woman with supernatural powers to make you believe a woman is floating in the air before you. He only needs to create an unusual setting capable of exploiting your implicit knowledge and perceptual skills. Similarly, the "illusory" appearance of the colors of afterimages is not caused by their illusory nature, but rather by their strangeness ${ }^{34}$ in relation to the perceiver's previous experiences. Harry Houdini once said, "Never try to fool children, they expect nothing, and therefore see everything." Afterimages show us that, unlike children, we are easily fooled when the reality we perceive departs from the reality we are used to perceiving.

Acknowledgements An early version of this paper was presented at the annual Philosophy of Science Conference workshop held at the Inter-University Centre in Dubrovnik in in April 2016. I'm grateful to Manuel García-Carpintero, Mohan Matthen and Kevin Mulligan for helpful discussions and encouragement. I would also like to thank two anonymous referees for their insightful comments and questions.

Funding Open access funding provided by University of Geneva.

Open Access This article is licensed under a Creative Commons Attribution 4.0 International License, which permits use, sharing, adaptation, distribution and reproduction in any medium or format, as long as you give appropriate credit to the original author(s) and the source, provide a link to the Creative Commons licence, and indicate if changes were made. The images or other third party material in this article are included in the article's Creative Commons licence, unless indicated otherwise in a credit line to the material. If material is not included in the article's Creative Commons licence and your intended use is not permitted by statutory regulation or exceeds the permitted use, you will need to obtain permission directly from the copyright holder. To view a copy of this licence, visit http://creativecommons.org/licen ses/by/4.0/.

\section{References}

Akins, K. 2014. Black and White and Colour n R. Brown (ed.) Consciousness inside and out: Phenomenology, neuroscience and the nature of experience. Springer Verlag.

Allen, K. 2009. Inter-species variation in colour perception. Philosophical Studies 142: 197-220.

Allen, K. 2010. In defence of natural daylight. Pacific Philosophical Quarterly 91 (1): 1-18.

Austin, J.L. 1962. Sense and Sensibilia. Oxford: Oxford University Press.

Berkeley, G.1734. Three dialogues between Hylas and Philonous.

Block, N. 1996. Mental paint and mental latex. In Philosophical issues 7, ed. E. Villanueva, 19-49. Atascadero CA: Ridgeview Publishing Company.

Boghossian, P.A., and J.D. Velleman. 1989. Colour as secondary quality. Mind 98 (389): 81-103.

Byrne, A., and D. Hilbert. 2003. Color realism and color science. Behavioral and Brain Sciences 26: 791-794.

\footnotetext{
34 For a defense of the view that familiarity with the conditions of perceptual experiences is crucial in determining whether or not a given appearance is misleading, see Genone (2014) and Mizrahi (2019).
} 
Campbell, K. 1979. The implications of Land's theory of color vision. In Logic, methodology, and philosophy of science VI : Proceedings of the sixth international congress of logic, methodology, and philosophy of science, Hannover, ed. L.J. Cohen et al., 541-552.

Campbell, J. 2002. Reference and consciousness. Oxford: Oxford University Press.

Chisholm, R. 1957. Perceiving: A philosophical study. Cornell: Cornell University Press.

Churchland, P. 2005. Chimerical colors: Some phenomenological predictions from cognitive neuroscience. Philosophical Psychology 18 (5): 527-560.

Crowther, T., and C. Mac Cumhaill. 2018. Perceptual Ephemera. Oxford: Oxford University Press.

Fish, W. 2009. Perception, hallucination, and illusion. Oxford: Oxford University Press.

French, C. 2014. Naive realist perspectives on seeing blurrily. Ratio 27: 393-413.

Genone, J. 2014. Appearance and illusion. Mind 490: 1-38.

Heider, Fritz. 1959. Thing and medium. Psychological Issues I: 1-34.

Hilbert, David R. 1987. Color and color perception: A study in anthropocentric realism. Stanford: CSLI.

Jackson, F. 1977. Perception: A representative theory. Cambridge: Cambridge University Press.

Kalderon, M. 2007. Color Pluralism. Philosophical Review 116: 563-601.

Katz, D. 1935. The world of colour. London: Kegan.

Kind, A. 2008. How to believe in qualia. In The case for qualia, ed. E. Wright, 285-298. Cambridge, MA: MIT Press.

Knuth, P. 1891. Die Einwirkung der Bluthenfarben auf die photographische Platte. Botanische .Central Blätter 48: 161-165.

Logue, H. 2012. Why naive realism? Proceedings of the Aristotelian Society 112: 211-237.

MacLeod, D.I., and M. Hayhoe. 1974. Rod origin of prolonged afterimages. Science 185: 1171-1172.

Macpherson, Fiona. 2013. The Philosophy and Psychology of Hallucination: An Introduction. In Hallucination: Philosophy and Psychology, ed. F. Macpherson and D. Platchias. MIT Press.

Martin, M.G.F. 2002. The transparency of experience. Mind and Language 17: 376-425.

Martin, M.G.F. 2010. What's in a look? In Perceiving the world, ed. B. Nanay. Oxford: OUP.

Matthen, Mohan. 1999. The Disunity of Color. The Philosophical Review 108 (1): 47-84.

Mizrahi, V. 2006. Colour objectivism and colour pluralism. Dialectica 60 (3): 283-306. https://doi.org/10. 1111/j.1746-8361.2006.01070.x.

Mizrahi, V. 2010. Colour and transparency. Rivista di Estetica 43: 1. https://doi.org/10.4000/estetica.1804.

Mizrahi, V. 2019. Mirrors and Misleading Appearances. Australasian Journal of Philosophy 97 (2): 354-367. https://doi.org/10.1080/00048402.2018.1481119.

Moore, G.E. 1942. A reply to my critics. In The philosophy of G. E. Moore, ed. P.A. Schilpp, 535-677. Evanston ILL: Northwestern University Press.

Noë, A. 2004. Action in perception. Cambridge, MA: The MIT Press.

O'Shaughnessy, B. 2000. Consciousness and the world. Oxford: OUP.

Phillips, I. 2013. Afterimages and sensation. Philosophy and Phenomenological Research 87: 417-453.

Shevell, S.K., R. St Clair, and S.W. Hong. 2008. Misbinding of color to form in afterimages. Visual Neuroscience 25: 355-360.

Shimojo, S., Y. Kamitani, and S. Nishida. 2001. Afterimage of perceptually filled-in surface. Science. 293: 1677-1680.

Siegel, S. 2010. The contents of visual experience. Oxford: New York.

Smart, J.J.C. 1963. Philosophy and scientific realism. London: Routledge.

Smith, A.D. 2002. The problem of perception. Cambridge, MA: Harvard University Press.

Sorensen, R. 2008. Seeing dark things. Oxford: Oxford University Press.

Thompson, E., A. Palacios, and F.J. Varela. 1992. Ways of coloring: Comparative color vision as a case study for cognitive science. Behavioral and Brain Sciences 15: 1-74.

Virsu, V., and P. Laurien. 1977. Long-lasting afterimages caused by neural adaptation. Vision Research 17 (7): $853-860$.

Wright, B. 2012. Darkness visible? Australasian Journal of Philosophy 90: 39-55.

Zaidi, Qasim. 2001. Color Constancy in a rough world. Color Research and Application 26: S192-S200.

Zaidi, Q., R. Ennis, D. Cao, and B. Lee. 2012. Neural locus of color afterimages. Current Biology 22: 220-224. https://doi.org/10.1016/j.cub.2011.12.021.

Publisher's Note Springer Nature remains neutral with regard to jurisdictional claims in published maps and institutional affiliations. 